\title{
WFUMB position paper on reverberation artefacts in lung ultrasound: B-lines or comet-tails?
}

\author{
Gebhard Mathis ${ }^{1}$, Rudolf Horn ${ }^{2}$, Susanne Morf ${ }^{2}$, Helmut Prosch ${ }^{3}$, Serena Rovida ${ }^{4}$, \\ Gino Soldati ${ }^{5}$, Beatrice Hoffmann ${ }^{6}$, Michael Blaivas ${ }^{7}$, Christoph F Dietrich ${ }^{8}$
}

${ }^{1}$ Rankweil, Austria, ${ }^{2}$ Center da sandà Val Müstair, Switzerland, ${ }^{3}$ Department of Biomedical Imaging and Image-guided Therapy, Medical University Vienna, Austria, ${ }^{4}$ Department of Emergency Medicine, Royal London Hospital, Barts Health NHS Trust, United Kingdom, ${ }^{5}$ Diagnostic and Interventional Ultrasound Unit, Valle del Serchio General Hospital, Lucca, Italy, ${ }^{6} \mathrm{M}$. Department of Emergency Medicine, Beth Israel Deaconess Medical Center, Harvard Medical School, Boston, USA, ${ }^{7}$ University of South Carolina School of Medicine, Department of Emergency Medicine, St Francis Hospital, Columbus Georgia, USA, ${ }^{8}$ Hirslanden Kliniken Bern, Beau Site, Salem and Permanence, Bern, Switzerland

\begin{abstract}
The analysis of vertical reverberation artefacts is an essential component of the differential diagnosis in pulmonary ultrasound. Traditionally, they are often, but not exclusively, called B-line artefacts (BLA) and/or comet tail artefacts (CTA), but this view is misleading.

In this position paper we clarify the terminology and relation of the two lung reverberation artefacts BLA and CTA to specific clinical scenarios. BLA are defined by a normal pleura line and are a typical hallmark of cardiogenic pulmonary edema after exclusion of certain pathologies including pneumonia or lung contusion, whereas CTAs show an irregular pleura line representing a variety of parenchymal lung diseases. The dual approach using low frequency transducers to determine BLA and high frequency transducer to determine the pleural surface is recommended.
\end{abstract}

Keywords: lung ultrasound; artefact; B-lines; comet tails; guidelines; misdiagnosis

On clinical and experimental grounds, reverberation artefacts are heterogeneous artefacts whose nature is closely linked to the pleural and subpleural tissue composition [1-4]. These artifacts can be short or long, bright, smooth, well- or ill-defined, narrow or wide and different in shape (comet or laser-like) [5-7]. Hence, the word "Bline" is not sufficient to characterize the heterogenous presentation and the wide spectrum of these artifacts.

Consequently, it is not surprising that reverberation artefacts may appear differently in various pathologic

Received 08.11.2020 Accepted 28.11.2020

Med Ultrason

2021, Vol. 23, No 1, 70-73

Corresponding author: Prof. Dr. med. Christoph F. Dietrich, MBA

Department of Internal Medicine (DAIM)

Kliniken Hirslanden Bern, Beau Site,

Salem and Permanence, Schänzlihalde 11,

3031 Bern, Switzerland

E-mail: c.f.dietrich@googlemail.com conditions, depending on the nature of the subpleural process (e.g., edema, inflammation, infiltration, fibrosis) and on the transducer frequency, bandwidth and scanning format [5-7]. Additionally, recent experimental evidence provided more information regarding the origins of Bline artefacts and how ultrasound settings should be adjusted to optimize accuracy and precision when scanning the lung.

The aim of this position paper is to summarize the current best available definition of the two vertical pulmonary reverberation artefacts: B-line artefact (BLA) and comet tail artefact (CTA), to improve diagnostic accuracy and specificity, clinical management and clinical communication.

In a seminal publication, Lichtenstein et al presented the comet-tail artefact (CTA) in lung ultrasound as sign allowing bedside distinction between pulmonary edema and chronic obstructive lung disease. The authors distin- 


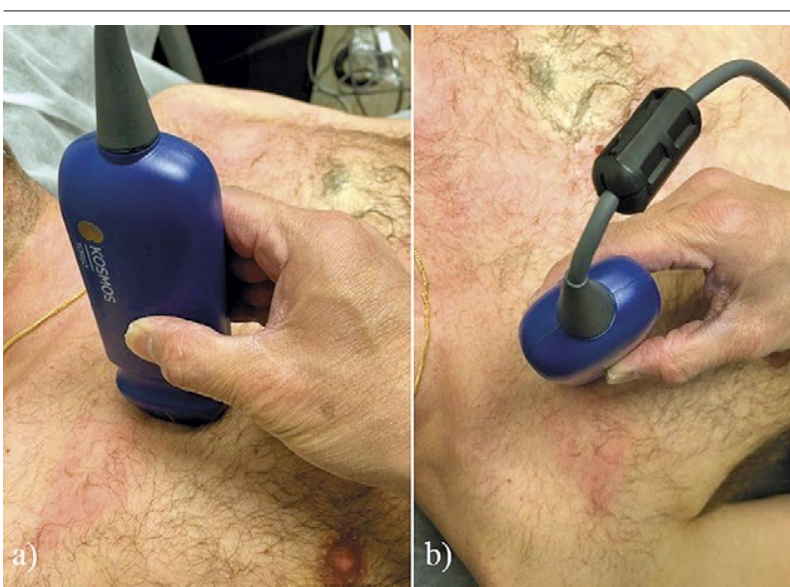

Fig 1. Shows proper transducer orientation ( $a$ and $b$ ) allowing for minor angle adjustment so the ultrasound beam can intersect the pleura perpendicularly, achieving the best artifacts. Unknowingly performing off axis scanning can mislead the provider regarding artifact presence or absence.

guished between a long and short type of CTA and stated that only the long type was diagnostic for the so-called interstitial syndrome [8]. The thickening of interlobular septa, at the points of greatest impedance, was considered the likely explanation for these artifacts [8]. However, this initial description lacks the very relevant information provided by high-resolution ultrasound of the lung surface to exclude changes of the pleura line and the distinct analysis of the shape of the artefact. Such highresolution images are possible with modern ultrasound equipment including broad frequency linear transducers. The rudimentary ultrasound equipment utilized by the authors lacked modern day ultrasound transducer capability and resolution; the low resolution, micro-convex transducer utilized significantly limited any analysis of the pleura [8].

An international consensus conference in 2012 defined BLA as discrete, laser-like vertical hyperechoic reverberation artifacts that arise from the pleural line and extend to the bottom of the screen without fading, moving synchronously with lung sliding. Multiple BLA were defined as the sonographic sign of a so called "interstitial lung syndrome". A positive region was defined by the presence of three or more BLA in a longitudinal plane between two ribs [4]. The consensus conference statement omits discussion of additional information yielded by high resolution ultrasound interrogation of the lung surface including excluding changes of the pleura and distinct analysis of the shape of the artefact. In addition, the consensus paper not only focused on edema but also on other pathophysiological disorders of the lung parenchyma including pulmonary fibrosis and interstitial pneumonia, which the authors referred to as "interstitial syndrome".
Since the publication of the consensus statement, some authors extended the term "focal interstitial syndrome" to include other disorders such as lung contusion, pneumonia or pulmonary embolism. In this context, all vertical reverberation artefacts radiating from the pleura into the lung parenchyma were designated BLA. An accurate analysis of the various published descriptions of BLA shows undifferentiated, mixed, and often contradictory conclusions $[9,10]$. The imprecise use of the terms BLA and CTA in lung US have led to confusion, which is seen in routine daily practice as well as published literature $[11,12]$. A more precise definition of the spectrum of reverberation artefacts may be helpful to better differentiate cardiogenic pulmonary edema from parenchymal lung diseases $[3,6,13]$. Therefore, parenchymal lung diseases have to be excluded before a BLA analysis for cardiogenic pulmonary edema is undertaken.

\section{Suggested approach:}

The transducer should be positioned such that the emenating ultrasound beam perpendicularly intersects the surface of the lung to maximize likelihood of seeing all BLA and CLA as well as A line artifacts (fig 1). A recent study highlighted the potentially detrimental effects of placing the focal zone below the pleural line, using spatial compounding, higher frequency and tissue harmonics [14]. Once machine settings and transducer orientation have been optimized, we suggest that two most important and distinct vertical lung artefacts should be differentiated: BLA and CTA. While true BLA (fig 2) originate from a smooth pleural reflex due to cardiogenic pulmonary edema and present in a diffuse pattern, CTAs are seen in many lung disorders with irregular and fragmented pleural reflexes and can be focal or diffuse (fig 3). Hence, the initial step should be to determine if there is evidence for diffuse pulmonary disease or defined focal or localized pathology. Focal lung pathologies by definition should display vertical artifacts that are consistent with CTAs (fig 4).

Diffusely distributed vertical reverberation artefacts can be divided into two groups: with or without detectable pleural line irregularities and with stable or distally widening width:

1 . The reverberation artefact (evaluated by low frequency transducer $<5 \mathrm{MHz}$ without interfering presets) is called BLA if arising from a smooth pleural line (evaluated by high frequency transducer $\geq 10 \mathrm{MHz}$ ). The BLA arises from edema within the interstitium, is well defined with stable width, hyperechoic and extending indefinitely (the entire depth, at least $10 \mathrm{~cm}$ ), erasing A-lines and moving with lung sliding. It is important to realize that many modern ultrasound machines have post-processing and other features which will 


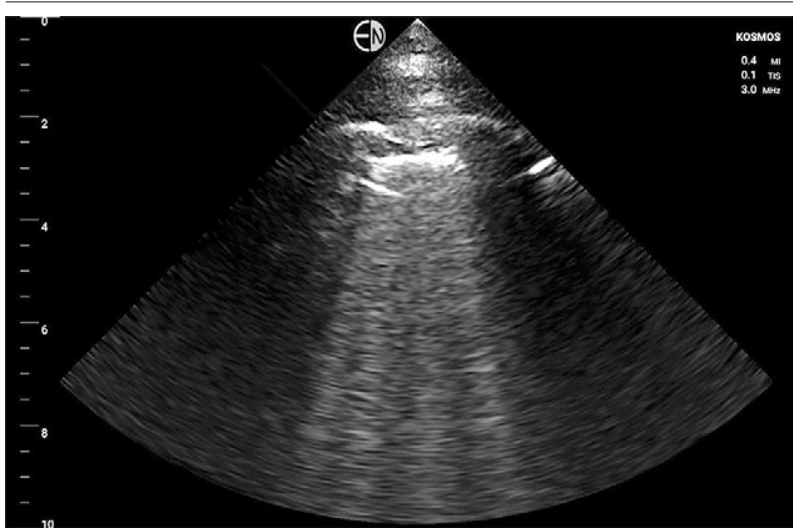

Fig 2. True BLA originate from a smooth pleural reflex due to cardiogenic pulmonary edema and present in a diffuse pattern as seen in this example.

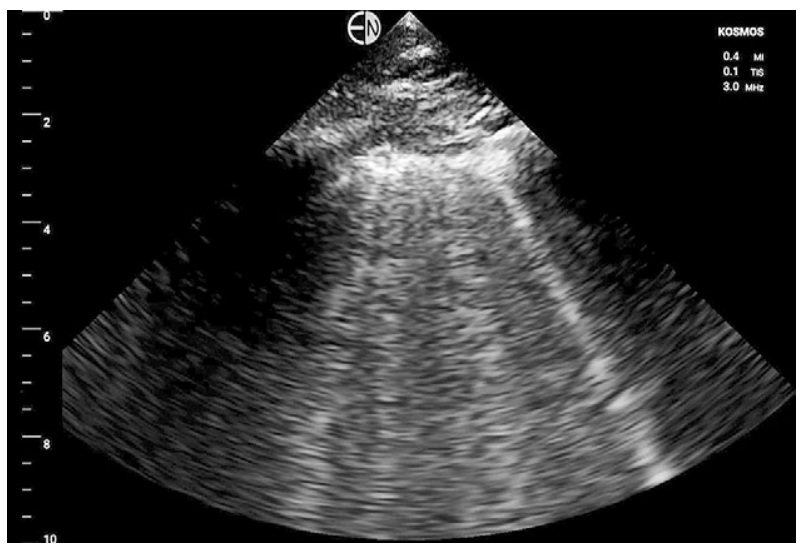

Fig 3. An example of CTAs seen in a patient with pulmonary fibrosis. Note the detectable pleural line irregularities seen.

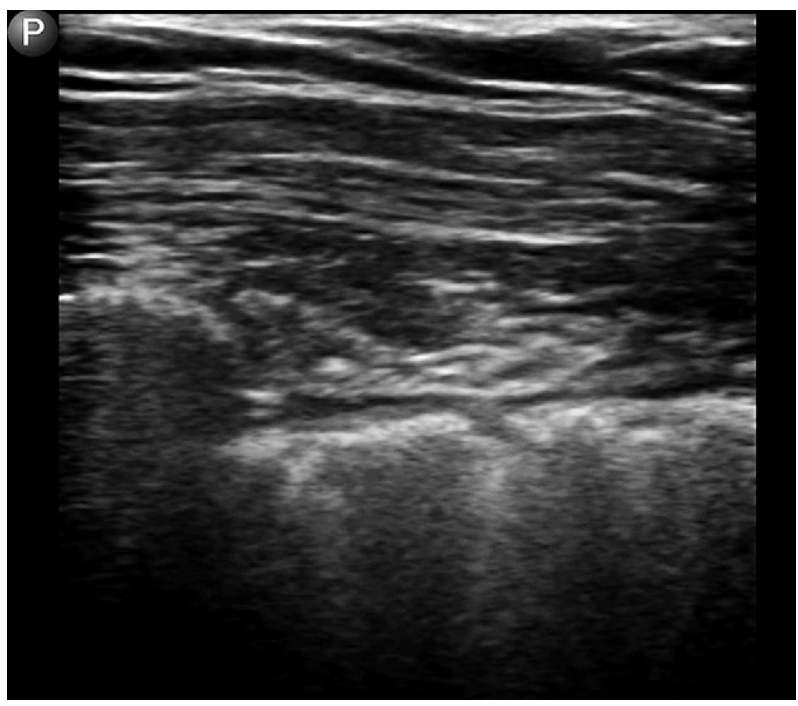

Fig 4. An example of focal lung pathologies, which would give rise to vertical CTAs in a patient with pulmonary fibrosis. Detectable pleural line irregularities are obviously seen with the higher transducer frequency. eliminate not only BLA but essentially all discernable image detail near the bottom of the screen at greater depths (fig 5).

2. The reverberation artefact is called CTA if arising from an irregular (or fragmented) pleural line (evaluated by high frequency transducer $\geq 10 \mathrm{MHz}$ ), changes in width (such as e a comet with narrow head and wide tail), is well defined, hyperechoic, and extending definitely $(<10 \mathrm{~cm}$ in depth) (evaluated by low frequency transducer $<5 \mathrm{MHz}$ without interfering presets). It is important to make sure image compounding is turned off to make sure the CTA is not distorted farther field [14].

The differentiation of BLA from CTA is also dependent on the technical adjustments of several external factors, including the type of ultrasound machine, transducers and probe frequencies [6].

In conclusion, the correct diagnosis of pulmonary edema (the etiology of which may be decided upon through integration of ultrasound data with clinical presentation) in the emergency setting is crucial for the correct management of the patient. The differentiation between ultrasonographic BLA and CTA, using two types (high and low frequency) of transducers allows accurate differentiation between pulmonary edema and other cause of diffuse pulmonary pathology. Both can lead to acute respiratory failure but may require different clinical management. Localized pulmonary diseases representing with CTA are distinguished. Mixed forms of diffuse, but also diffuse and focal lung diseases have to be considered.

\section{Conflict of interest: none}

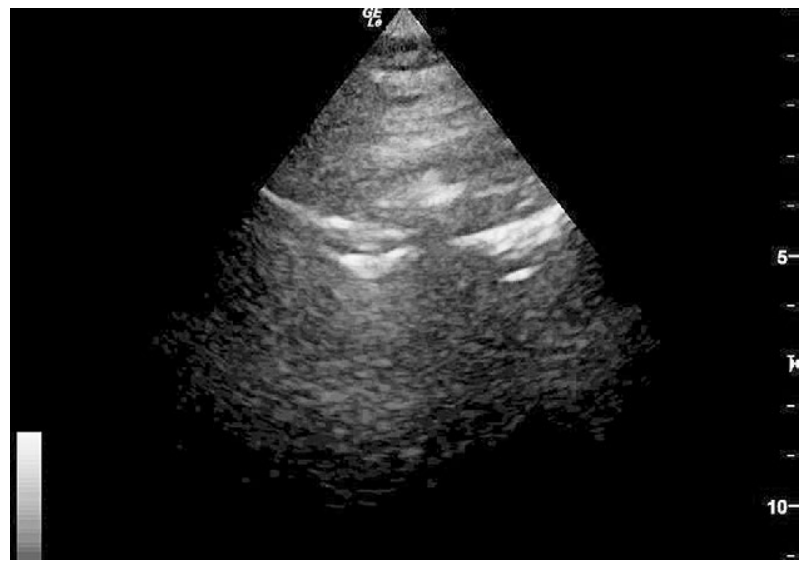

Fig 5. An example of modern ultrasound machine image processing eliminating not only BLA or CTAs but all image features at in the far field. The bright pleural line is seen in the near field but nearly the entire image drops out in the lower third of the screen. 


\section{References}

1. Soldati G, Smargiassi A, Inchingolo R, et al. Lung ultrasonography may provide an indirect estimation of lung porosity and airspace geometry. Respiration 2014;88:458-468.

2. Soldati G, Giunta V, Sher S, Melosi F, Dini C. "Synthetic" comets: a new look at lung sonography. Ultrasound Med Biol 2011;37:1762-1770.

3. Soldati G, Copetti R, Sher S. Sonographic interstitial syndrome: the sound of lung water. J Ultrasound Med 2009;28:163-174.

4. Volpicelli G, Elbarbary M, Blaivas M, et al. International evidence-based recommendations for point-of-care lung ultrasound. Intensive Care Med 2012;38:577-591.

5. Soldati G, Demi M, Smargiassi A, Inchingolo R, Demi L. The role of ultrasound lung artifacts in the diagnosis of respiratory diseases. Expert Rev Respir Med 2019;13:163-172.

6. Dietrich CF, Mathis G, Blaivas M, et al. Lung B-line artefacts and their use. J Thorac Dis 2016;8:1356-1365.

7. Dietrich CF, Mathis G, Blaivas M, et al. Lung artefacts and their use. Med Ultrason 2016;18:488-499.

8. Lichtenstein D, Meziere G, Biderman P, Gepner A, Barre O. The comet-tail artifact. An ultrasound sign of alve- olar-interstitial syndrome. Am J Respir Crit Care Med 1997; 156:1640-1646.

9. Soldati G, Demi M. The use of lung ultrasound images for the differential diagnosis of pulmonary and cardiac interstitial pathology. J Ultrasound 2017;20:91-96.

10. Soldati G, Demi M, Demi L. Ultrasound patterns of pulmonary edema. Ann Transl Med 2019;7(Suppl 1):S16.

11. Pivetta E, Goffi A, Nazerian P, et al. Lung ultrasound integrated with clinical assessment for the diagnosis of acute decompensated heart failure in the emergency department: a randomized controlled trial. Eur J Heart Fail 2019;21:754766.

12. Yue Lee FC, Jenssen C, Dietrich CF. A common misunderstanding in lung ultrasound: the comet tail artefact. Med Ultrason 2018;20:379-384.

13. Dietrich CF, Mathis G, Cui XW, Ignee A, Hocke M, Hirche TO. Ultrasound of the pleurae and lungs. Ultrasound Med Biol 2015;41:351-365.

14. Kameda T, Kamiyama N, Kobayashi H, Kanayama Y, Taniguchi N. Ultrasonic B-Line-Like Artifacts Generated with Simple Experimental Models Provide Clues to Solve Key Issues in B-Lines. Ultrasound Med Biol 2019;45:16171626. 